\title{
Philosophiques
}

\section{Les générations, le fleuve et l'océan}

\section{Axel Gosseries}

Volume 42, numéro 1, printemps 2015

URI : https://id.erudit.org/iderudit/1032222ar

DOI : https://doi.org/10.7202/1032222ar

Aller au sommaire du numéro

\section{Éditeur(s)}

Société de philosophie du Québec

\section{ISSN}

0316-2923 (imprimé)

1492-1391 (numérique)

Découvrir la revue

\section{Citer cet article}

Gosseries, A. (2015). Les générations, le fleuve et l'océan. Philosophiques, 42(1), 153-176. https://doi.org/10.7202/1032222ar

\section{Résumé de l'article}

À la suggestion de Jefferson, nous nous proposons de prendre au sérieux la comparaison entre nations et générations dans le cadre d'une théorie philosophique de la justice et de la démocratie préoccupée par nos devoirs envers les membres d'autres générations. Nous nous concentrons ici sur trois des caractéristiques propres aux relations intergénérationnelles, à travers une comparaison avec des situations internationales spécifiques. La première a trait à l'immobilité temporelle des personnes au delà de la période s'étendant de leur naissance à leur mort. Cette immobilité rend certaines ressources qui sont spécifiques à des périodes données, inaccessibles aux générations des autres périodes, ce qui peut importer pour certaines théories de la justice. Nous nous intéressons ensuite au caractère enclavé de chaque génération. La transmission intergénérationnelle des biens dépend alors de la collaboration tant passive qu'active des générations de transit. Nous montrons en quoi cela importe pour la définition de nos obligations de justice intergénérationnelle. Enfin, nous insistons sur le caractère unidirectionnel de l'écoulement du temps - cette fois à travers l'analogie avec les nations riveraines d'un fleuve - et son effet sur la distribution temporelle des coûts et bénéfices, et des vulnérabilités. Nous en examinons les implications normatives possibles pour la définition de la juste distribution du pouvoir entre générations. À travers ces trois illustrations, nous espérons montrer la pertinence de cette approche anatomico-analogique pour la compréhension de ce que nous devons aux autres générations, et en particulier aux générations futures.
Ce document est protégé par la loi sur le droit d'auteur. L’utilisation des services d’Érudit (y compris la reproduction) est assujettie à sa politique d'utilisation que vous pouvez consulter en ligne.

https://apropos.erudit.org/fr/usagers/politique-dutilisation/ 


\title{
Les générations, le fleuve et l'océan
}

\author{
AXEL GOSSERIES
}

Fonds de la recherche scientifique (FNRS)

Université de Louvain (UCL, Chaire Hoover d'éthique économique et sociale)

\section{"[...] L'on dessine mal parce que l'on représente ce que l'on sait au lieu de représenter ce que l'on voit.»}

(R. HAINARD, 1990)2

\begin{abstract}
RÉSUMÉ. - À la suggestion de Jefferson, ${ }^{3}$ nous nous proposons de prendre au sérieux la comparaison entre nations et générations dans le cadre d'une théorie philosophique de la justice et de la démocratie préoccupée par nos devoirs envers les membres d'autres générations. Nous nous concentrons ici sur trois des caractéristiques propres aux relations intergénérationnelles, à travers une comparaison avec des situations internationales spécifiques. La première a trait à l'immobilité temporelle des personnes au delà de la période s'étendant de leur naissance à leur mort. Cette immobilité rend certaines ressources qui sont spécifiques à des périodes données, inaccessibles aux générations des autres périodes, ce qui peut importer pour certaines théories de la justice. Nous nous intéressons ensuite au caractère enclavé de chaque génération. La transmission intergénérationnelle des biens dépend alors de la collaboration tant passive qu'active des générations de transit. Nous montrons en quoi cela importe pour la définition de nos obligations de justice intergénérationnelle. Enfin, nous insistons sur le caractère unidirectionnel de l'écoulement du temps - cette fois à travers l'analogie avec les nations riveraines d'un fleuve - et son effet sur la distribution temporelle des coûts et bénéfices, et des vulnérabilités. Nous en examinons les implications normatives possibles pour la définition de la juste distribution du pouvoir entre générations. À travers ces trois illustrations, nous espérons montrer la pertinence de cette approche anatomicoanalogique pour la compréhension de ce que nous devons aux autres générations, et en particulier aux générations futures.
\end{abstract}

ABSTRACT. - Following Jefferson's suggestion, I take seriously the promises of a comparison between nations and generations, as part of a philosophical

1. Axel Gosseries est maître de recherches au Fonds de la recherche scientifique (FNRS), Professeur à l'Université de Louvain (UCL, Chaire Hoover d'éthique économique et sociale) et Franz Weyr Fellow de l'Académie tchèque des sciences (CeLAPA). Il tient à remercier chaleureusement de nombreux collègues pour leurs suggestions sur des versions antérieures de ce texte, les audiences qui ont assisté à la présentation de ces versions antérieures (Yerevan, Madrid et Besançon en 20I4; Braga en 20I3; Montpellier, Barcelone, Genève et Bucarest en 20I 2) ainsi que deux évaluateurs anonymes de la revue. La rédaction de ce texte a bénéficié des financements liés aux projets ARC 09/I4-O I 8 «sustainability " (Communauté française de Belgique) et ESF ENRI- «Rights to a green future».

2. Crispini $(1996,94)$ (cité in)

3. "We seem not to have perceived that, by the law of nature, one generation is to another as one independent nation to another " (Jefferson to Madison, Sept 6, I789). 
theory of justice and of democracy concerned with our obligations towards members of other generations. I focus here on three of the features that are specific to intergenerational relations, through a comparison with corresponding international situations. The first feature has to do with people's temporal immobility beyond the lapse of time that extends from their birth to their death. Such immobility renders some period-specific resources inaccessible to generations from other periods, which may have implications for some theories of justice. The second feature that I look into is the period-locked nature of the members each generation. The intergenerational transfer of goods to future generations then depends on the passive as well as active collaboration of transit generations. I show why and to what extent this matters to the definition of our intergenerational obligations. Finally, I insist on the one-directional feature of time's passage - through an analogy with the situation of riparian nations along a river - and on its effect on the temporal distribution of costs and benefits, as well as of vulnerabilities. I explore the potential normative implications of such one-directionality for the definition of a fair distribution of power between generations. Through looking at these three features and closely examining their implications, I also hope to stress the relevance of an anatomicoanalogical method for the understanding of what we owe other generations, and especially future ones.

\section{Introduction}

I. Pour qui se penche philosophiquement sur les questions de justice intergénérationnelle, qui s'interroge sur ce que nous devons aux générations à venir, une des tâches à entreprendre pour asseoir la robustesse d'une proposition de principe consiste à rechercher des angles de vue différents et à la confronter avec ceux-ci. Il s'agit non seulement d'identifier - fût-ce axiomatiquement - des théories normatives alternatives, mais aussi - de manière partiellement indépendante - , de tenter un autre décryptage anatomique de la nature du problème. Une méthode possible consiste à se pencher sur une analogie entre la sphère intergénérationnelle et le domaine international. La comparaison est souvent évoquée, mais trop rarement approfondie ${ }^{4}$.

C'est cette comparaison et ses implications que nous voudrions explorer ici, non pas de manière systématique, mais à travers trois illustrations spécifiques. La philosophie peut d'ailleurs tirer parti sur ce point du regard posé sur la dimension internationale par d'autres disciplines comme le droit international ou l'économie géographique qui ont développé des concepts centrés sur l'espace géographique. Des outils, des propositions qui peuvent ensuite être testés analogiquement dans le champ intergénérationnel, mettant éventuellement en lumière des caractéristiques propres à la dimension temporelle. Nous n'examinerons pas directement ici comment le droit des fleuves, le droit de la mer ou le droit de la guerre, par exemple, nous aident à comprendre, en passant de l'espace au temps, la structure des questions

4. Pour une exception, voir le travail précurseur de Barry (I979). 
intergénérationnelles. Nous ne nous pencherons pas non plus sur la manière dont l'économie géographique évalue la dimension des coûts de transport et de stockage ou les effets économiques d'une géographie enclavée. Ce que nous allons proposer se situe en amont d'un tel travail et vise à en proposer en quelque sorte les prolégomènes, et à le faire de manière sélective, sur base de trois exemples. Il s'agit de mettre en regard générations et nations sous un angle bien particulier, celui de leur relation respective au territoire et à la période.

2. Pour les besoins du présent exercice, et en particulier de la première des comparaisons que nous proposerons, définissons chacune de ces deux notions de manière très spécifique:

Génération: Ensemble des personnes nées au cours d'une même période

Nation: Ensemble des personnes nées sur un même territoire

Si la définition de "génération » correspond à ce que les démographes nomment "cohorte de naissance ", celle de "nation » - qui vise à la rapprocher le plus possible de celle de "génération» - est assez éloignée du sens plus riche privilégié par les sciences sociales. Le lien fort de la nation au territoire de naissance dans la présente définition - qui renvoie à la connotation de l'anglais «native» - est évidemment discutable. Il a pour seule raison d'être de nous centrer sur ce qui est essentiel à la comparaison entre les dimensions du «territoire» (nations) et de la "période» (générations). La nationalité, comme la "générationalité », est donc déterminée dès la naissance et pour toujours. Cette définition de "nation" ne permet ainsi ni à plusieurs nations de partager un même territoire de naissance, ni à une même nation de partager plusieurs territoires de naissance, ni à un individu de changer de nationalité. Cela est cependant compatible avec le fait que tous les territoires soient multinationaux en cas de migrations importantes. Cette approche ne serait totalement trompeuse que si l'idée de nation telle qu'utilisée dans le terme «international» n'entretenait aucune relation avec l'idée de territoire, ce qui n'est pas le cas et ce qui ne préjuge bien sûr en rien de notre position sur la question de l'État-nation, et encore moins sur celle d'un prétendu «droit du sol $»^{5}$.

3. Le chemin emprunté ici se penchera sur deux types d'espaces internationaux: les fleuves et les océans. Nous nous demanderons d'abord s'il n'est pas vrai que les générations connaissent une situation analogue à celle de nations insulaires dépourvues de moyens de navigation en haute mer comme des caravelles (sect. I). Ensuite, les générations ne se trouvent-elles pas aussi dans une situation comparable à celles d'États enclavés, sans accès direct à la mer? (sect. 2). Enfin, ne peut-on mettre en regard l'écoulement du 
temps et l'écoulement de l'eau dans le lit d'un fleuve? Les générations ne devraient-elles pas alors entretenir entre elles des relations analogues à celles que devraient entretenir des pays d'amont et d'aval partageant l'usage d'un même cours d'eau (Sect. 3).

Pour chacune de ces trois comparaisons, nous nous efforcerons d'identifier les implications normatives des spécificités intergénérationnelles ainsi mises en lumière. Car décaler le regard, procéder à un travail "anatomique " nourri par l'analogie ne suffit pas. Ce travail nous importe d'abord dans la mesure où il nous permettrait d'identifier, au plan normatif, des implications qui seraient sinon restées invisibles. Insistons sur le fait que nous pointerons ainsi trois caractéristiques qui sont générales aux relations intergénérationnelles (immobilité des personnes, enclavement et direction du temps) alors qu'elles sont limitées à des contextes spécifiques en relations internationales. De plus, si ces caractéristiques sont bien générales au domaine intergénérationnel, elles n'affectent cependant pas nécessairement à chaque fois l'ensemble des biens composant le panier à transmettre d'une génération à l'autre. Enfin, répétons-le, nous ne prétendons pas à ce stade procéder à une comparaison complète entre relations internationales et relations intergénérationnelles.

4. Il y a une autre caractéristique importante que nous laisserons ici de côté, celle de l'engendrement, de la genèse. Le terme "génération » renvoie plus souvent dans l'usage au fruit de l'acte de mise au monde qu'à cet acte luimême. Pourtant, ce dernier est certainement un caractère distinctif des relations intergénérationnelles: les occupants d'une période sont directement et causalement responsables de l'existence des occupants de la période suivante par le fait de la natalité. Ce n'est pas le cas des résidents d'un territoire par rapport à ceux d'un autre. Le Canada, par exemple, n'est pas en mesure de décider seul de l'existence et de la taille de la prochaine génération d'Haïtiens, même s'il peut l'influencer par ses politiques migratoires. L'existence même de la génération qui nous suit est le fruit de notre volonté procréatrice. Et si cette dimension singulière a un lien avec la flèche du temps nous ne sommes pas responsables de l'existence de nos ancêtres -, elle en est aussi distincte.

Une question passionnante est alors de savoir si le fait de causer l'existence même de la génération suivante modifie la nature et/ou l'intensité de nos obligations à leur égard. On pourrait penser qu'elle en renforce l'intensité. C'est peut-être le cas si l'on partage l'intuition selon laquelle tout problème qu'aurait à affronter la génération suivante serait en quelque sorte «causé » par nous, par le fait d'avoir engendré l'existence même des personnes concernées. C'est peut-être le cas aussi pour une théorie distributive qui voudrait faire peser sur la génération procréatrice les coûts liés au fait de donner naissance à une génération nouvelle beaucoup plus grande ou beaucoup plus petite que la nôtre. 
Pourtant, cette même relation d'engendrement est aussi susceptible et ce de manière inattendue - d'affaiblir plutôt que de renforcer nos obligations envers le futur. Ce serait vrai dans la mesure où des conséquences problématiques pour le futur seraient associées à l'acte procréatif et constitueraient la condition nécessaire de l'existence d'individus spécifiques. On peut en effet avancer l'hypothèse que, dans un certain nombre de cas, les politiques environnementales par exemple (ou leur absence) ont aussi un impact sur... le moment de nos actes procréatifs et donc aussi sur... l'identité des personnes ainsi conçues. Ce constat, qui peut paraître tiré par les cheveux, a trait à ce que les philosophes qualifient de problème de la «nonidentité $^{6}$ ». Il ne nous sera malheureusement pas possible de l'aborder ici. La littérature sur ce sujet est considérable - et souvent répétitive. Mais cela constitue une raison supplémentaire possible pour laquelle la dimension d'engendrement serait porteuse d'implications normatives différentes de la migration ou de l'invasion de l'espace des nations.

\section{Mobilité temporelle réduite et inaccessibilité de ressources extra- périodiques}

5. Entamons donc notre périple par une première illustration. Repartons pour ce faire de notre définition des nations et des générations. Un élément essentiel a trait à la mobilité des personnes. Si l'on prend au sérieux nos deux définitions ci-dessus, nul ne peut changer de génération (ou de "générationalité »), ni de nationalité. Chacun de nous est à jamais attaché à son territoire de naissance et à sa période de naissance. Certes, même avec notre définition étroite de "nation ", il reste possible de quitter son territoire de naissance, même si cela n'impliquera aucun changement de nationalité. Si une génération n'est pas, elle non plus, contrainte de rester dans sa période de naissance, elle voit malgré tout son existence enserrée dans une période plus large comprise entre sa naissance et sa mort. Il n'y a pas de mobilité possible au delà de cette période. Il n'est pas possible de parcourir des périodes antérieures à notre naissance ou postérieures à notre mort. Et même à l'intérieur de notre période d'existence, l'itinéraire de notre voyage temporel devra obligatoirement traverser les décennies successives dans leur ordre chronologique et pour une durée imposée. S’il est donc inexact de dire qu'il n'y a pas de voyage possible d'une sous-période à l'autre, la direction, l'ordre, le rythme de ce voyage ainsi que le fait même de voyager sont imposés. Il n'y a donc pas de mobilité libre dans le temps; et si mobilité il y a, elle est réduite aux frontières de notre existence.

Cela diffère du domaine des nations et des territoires, où, même si je ne peux changer de nation au sens défini plus haut, je peux décider de migrer ou non; et je peux décider jusqu'à un certain point vers quels territoires je 
veux migrer, à quelle fréquence, etc. Évidemment, les limites de ma naissance et de ma mort posent aussi une limite aux distances géographiques que je serai physiquement en mesure de parcourir. Mais il n'en reste pas moins qu'à l'échelle de la terre et d'une vie, il existe une différence entre mobilité spatiale et temporelle. L'on peut en réalité considérer qu'une génération est immobile au sens plus large où son existence sera limitée à un nombre limité de sous-périodes correspondant à son espérance de vie.

6. Ce qui nous intéresse, une fois cette analyse effectuée, c'est de dégager les conséquences normatives possibles de ce constat de mobilité temporelle réduite des personnes pour une théorie de la justice ${ }^{7}$. Une des théories pour lesquelles cela est susceptible de revêtir une certaine importance est le libertarisme de gauche ${ }^{8}$. Le raisonnement suivant vise à le montrer. Partons de Thomas Paine et de l'exemple d'un maillage de parcelles agricoles ${ }^{9}$. Pour Paine, les Terriens sont tous, conjointement, co-propriétaires au départ de ces parcelles dans leur état naturel. Par contre, ce n'est pas le cas des améliorations résultant des efforts de culture fournis par chacun d'entre nous. S'il est donc important de séparer les deux en ce qui concerne la justice, ils sont inséparables en pratique, considère Paine. Il propose donc un mécanisme par lequel, tout en acceptant l'idée de propriété, individuelle et exclusive, des terres cultivées et les inégalités qu'elle engendre, chaque propriétaire d'une parcelle devrait à l'ensemble de la société une somme correspondant à la rente foncière. Cette somme vise à couvrir la perte pour autrui liée au fait d'être dépossédé de la valeur des parcelles dans leur état non cultivé. Concrètement, Paine proposa à l'époque (I796) la mise en place d'un fonds alimenté par de tels paiements et par l'entremise duquel on verserait à chaque personne ayant atteint l'âge de $2 \mathrm{I}$ ans une somme de 5 livres «à titre de compensation partielle pour la perte de son héritage naturel résultant de l'introduction d'un système de propriété foncière ${ }^{10}$ ».

La clef consiste dans l'idée d'indemnisation pour la perte d'accès à la valeur naturelle des parcelles résultant de l'exclusion engendrée par l'appropriation privée. Dans le cas d'un maillage de parcelles agricoles, une série d'agriculteurs potentiels se disputent les différentes parcelles. La propriété privée implique l'exclusion d'autrui, et c'est cette exclusion qui prive les autres agriculteurs potentiels de la possibilité de cultiver les parcelles en question. Or nous devons tenter de faire en sorte qu'autrui ne se retrouve

7. Cette mobilité réduite, cette absence d'exit générationnel, a peut-être aussi des conséquences en théorie de la démocratie - comme le suggère un des évaluateurs anonymes. L'espace nous manque cependant pour les explorer ici

8. Voir par exemple Vallentyne et Steiner (2000).

9. Paine (I796).

10. Ibid. ( "as a compensation in part, for the loss of his or her natural inheritance, by the introduction of the system landed property»). 
pas dans une situation pire que dans l'état de nature, à savoir, pire qu'en l'absence d'autres personnes.

7. Pourtant, cela a-t-il du sens de dire qu'autrui me prive de la valeur d'une parcelle si de toute façon, même en son absence, je n'y aurais pas eu accès, étant incapable de l'atteindre par mes propres moyens? Cette question touche à un problème général pour les lockéens, que l'on peut décliner ici dans l'espace des générations pour certains types de biens. Si un bien n'est pas renouvelable tout en étant durable, sa consommation est rivale entre générations - au sens économique du terme. L'immobilité de chaque cohorte ne change rien dans ce cas: si une génération consomme ce bien, elle en prive une autre génération. La durabilité du bien neutralise ici l'immobilité de la génération. Par sa durabilité, le bien vient aux générations, même si les générations sont incapables d'aller à lui. On notera en outre qu'un bien non rival au plan intragénérationnel sera aussi non rival sur le plan intergénérationnel, même si sa durabilité n'est pas nécessairement garantie, comme dans le cas d'idées qui nécessiteraient un investissement éducatif pour leur préservation, faute de quoi elles seraient perdues.

Par contre, en ce qui concerne les biens éphémères ou les biens durables renouvelables, les choses sont différentes. Si un bien est éphémère et aura de toute façon disparu à la génération suivante, en le consommant, en en profitant, une génération ne prive aucune autre génération de ce bien. Les fluctuations dans le temps du climat naturel, de l'activité volcanique ou tectonique, ou des ressources en biodiversité font que certaines périodes sont plus riches que d'autres (court épisode climatique favorable, période de calme sismique, etc.). On peut donc parler de ressources fluctuantes ou éphémères. De même, si un bien est naturellement renouvelable, tant que je le consomme sans en dépasser le seuil de renouvellement, chaque génération qui en tire profit n'en prive aucune autre génération. Il importe donc de différencier les biens selon qu'ils sont durables, rivaux et non renouvelables, ou qu'ils sont éphémères ou durables renouvelables. Et il importe de bien comprendre la conjonction du constat d'immobilité générationnelle avec l'analyse de la nature de ces biens.

Avant d'aborder cette conjonction, insistons sur l'idée selon laquelle la situation de mobilité temporelle réduite est par exemple analogue à celle de pêcheurs de deux îles d'un même archipel incapables de naviguer en haute mer et de rejoindre d'autres îles, leur capacité de pêche étant limitée à la capture de poissons côtiers endémiques (équivalents spatiaux des ressources éphémères) ou aux poissons migrateurs océanodromes croisant leur île de temps à autre (équivalent spatiaux des ressources durables renouvelables). Le problème de rivalité ne se pose donc pas dans ce cas s'ils ne consomment que des poissons côtiers endémiques ou s'ils consomment des poissons migrateurs sans mettre en péril la capacité de renouvèlement du stock et le niveau de ce stock une fois qu'il aura atteint l'île suivante. 
8. Comment joindre l'analyse de la nature ${ }^{11}$ des biens (de leur «comportement » dans le temps) et celle de l'immobilité générationnelle ? On peut analyser la mobilité temporelle réduite comme une limitation dans les ressources internes des personnes (et des générations) affectant leur accès aux ressources externes "initialement» communes ${ }^{12}$. Les inégalités de ressources internes sont alors susceptibles d'affecter de trois manières très différentes le droit aux ressources externes des personnes, et ce en fonction de l'interprétation que l'on donne d'une approche lockéenne.

Selon la première option, que l'on qualifiera d' "anti-redistributive», l'on estime que ce qui est clef pour justifier mes obligations éventuelles envers autrui en matière de ressources externes est que mon usage d'une ressource prive autrui de l'usage de cette même ressource. Dans ce cas, on pourrait dire que les personnes qui ont des ressources internes moins fournies, et dont le handicap est tel qu'il réduit leur capacité à user des ressources externes, devraient recevoir une moindre part de la recette de la taxe sur la rente foncière telle que proposée par Paine. Ce serait un résultat particulièrement inégalitaire, couronnant la blessure d'insulte. Et c'est ce qui fait que d'aucuns pensent qu'une telle interprétation, même "de gauche", de la clause lockéenne reste tout à fait insuffisante. Un tétraplégique ne disposerait ainsi pas de compensations particulières pour ce qui est des droits sur la recette de la taxe de Paine, selon une telle approche. Pire, il verrait se réduire la part de cette recette à laquelle il aurait eu droit en étant valide. Cela cadrerait cependant avec la logique qui consiste à compenser les personnes pour ce dont on les prive, et uniquement pour cela.

La deuxième option, qui peut être qualifiée de «neutre ", consisterait à affirmer cette fois que la raison pour laquelle un utilisateur effectif doit indemniser autrui a trait au fait qu'il prive ce tiers de l'usage d'un bien qui lui appartenait (en co-propriété), et ce indépendamment de la capacité de ce tiers à en user personnellement sans l'aide d'autrui.

Quant à la troisième option, qu'on qualifiera de «redistributive», il s'agirait, à la suite par exemple d'Otsuka ${ }^{13}$, tout en respectant une forte protection de la propriété de soi (qui a trait aux ressources internes), d'allouer de manière inégalitaire le droit aux revenus des ressources externes afin de compenser les inégalités de ressources internes. L'on allouerait plus de droits sur les ressources externes à ceux qui sont les moins dotés sur le plan des ressources internes. Et on le ferait ainsi sans tomber dans des formes de taxation du travail incompatibles avec une forte protection de la propriété de

11. Nous laissons ici de côté la question importante du caractère culturel ou naturel d'une ressource.

12. Cela ne va pas de soi. La littérature sur le handicap attire notre attention sur le fait que le handicap est généralement à comprendre autant comme une inadéquation des individus à l'environnement que comme une inadéquation de l'environnement aux individus.

13. Otsuka (2003). 
soi. Dans ce cas, il se peut qu'une tétraplégique incapable d'accéder seule aux ressources externes concernées se voie octroyer une compensation plus importante que les autres en cas d'appropriation par un tiers. Il s'agit ainsi de dépasser Paine et d'allouer la recette de la taxe de Paine de manière inégalitaire, en accordant une part plus grande à ceux qui sont plus démunis pour ce qui est des ressources internes.

9. On le voit, en fonction du type d'interprétation lockéenne (antiredistributive, neutre ou redistributive), les différences de ressources internes, et en particulier celles qui affectent notre capacité à accéder à certaines ressources externes, se répercutent différemment. Il ne s'agit pas ici de départager ces différentes variantes. Cela peut être fait à la fois en se demandant quelle interprétation correspond le mieux à la logique centrale de l'approche lockéenne, mais aussi en s'interrogeant sur la question de savoir quelle interprétation cadre le mieux avec nos intuitions, formées de manière partiellement indépendante, relatives aux inégalités. L'objet de la présente section est plus circonscrit. Il s'agit seulement de montrer que le problème de mobilité temporelle réduite est susceptible d'avoir des conséquences normatives selon que l'on ait recours à l'une ou l'autre des versions de la théorie lockéenne.

ıo. Notons une complication supplémentaire. Si l'on peut analyser l'immobilité générationnelle comme un problème de ressources internes, cela n'implique pas nécessairement qu'il faille y voir un problème d'inégalité dans les ressources internes. Toutes les générations sont en effet également incapables d'accéder aux ressources éphémères spécifiques aux périodes qu'elles ne traverseront pas - même si les espérances de vie évoluent. On peut certes dire aussi qu'à chaque période seules les cohortes en vie sont capables d'accéder aux ressources éphémères spécifiques à une telle période, ce qui génère évidemment, à chaque moment, une capacité d'accès inégale par rapport aux générations antérieures ou ultérieures. Tout cela peut avoir son importance. Si l'on interprète la déficience interne de chaque génération comme égale, alors les interprétations neutre et redistributive de la clause lockéenne ne devraient pas conduire à des résultats différents, les ressources internes des personnes étant les mêmes. Par contre, la différence entre l'interprétation neutre et l'interprétation anti-distributive subsisterait, car la seconde ne verrait pas de raison de compenser les différences de ressources externes disponibles d'une période à l'autre, alors que la première serait capable de défendre une telle compensation.

I I. Ceci ouvre en réalité une interprétation nouvelle de la clause lockéenne intergénérationnelle. En effet, si une théorie lockéenne considère que je n'ai droit qu'aux ressources externes auxquelles j'aurais été capable d'avoir accès en l'absence de la génération précédente (mais pas des autres générations antérieures), il n'y a pas lieu de compenser des différences exogènes de ressources externes entre la période précédente et la période actuelle - par 
exemple des évolutions héritées ou naturelles dans les conditions climatiques. Une telle interprétation lockéenne devrait donc considérer que les fluctuations climatiques, sismiques, et autres, sources d'inégalités parfois très significatives entre les générations, ne doivent pas donner lieu à compensation, même si elles étaient prédictibles.

Une théorie égalitariste, non lockéenne, de la justice intergénérationnelle prendra par contre pleinement en compte ce genre de fluctuations, surtout si elles sont prédictibles dans une certaine mesure. En effet, pour un égalitariste, ce n'est pas le fait que mon usage prive autrui de cet usage qui importe et donne lieu à compensation. C'est l'arbitraire du caractère inégal des dotations qui justifie la redistribution.

Ce qui est intéressant, c'est qu'on peut imaginer une interprétation lockéenne allant dans cette direction également. C'est le cas si mon droit aux ressources s'étend aux ressources éphémères de périodes situées au-delà (avant ou après) de ma propre existence, s'il est indépendant de l'accessibilité de telles ressources/périodes pour moi. On peut alors imaginer une clause lockéenne intergénérationnelle qui, tout en restant centrée sur les ressources externes, exigerait de laisser à la génération suivante au moins autant de ressources externes, par exemple, que la moyenne entre le niveau de ressources (par tête) dont la génération présente aurait bénéficié en l'absence de la précédente, et dont la génération suivante aurait bénéficié en l'absence de la présente. À nouveau, il nous importe ici de montrer que l'accent sur la mobilité temporelle réduite permet de voir de telles différences, sans pour autant se prononcer ici sur la question de savoir laquelle de ces clauses lockéennes serait la plus authentiquement lockéenne, ni sur celle de savoir si c'est la bonne question à poser ${ }^{14}$.

I2. Concluons sur ce premier point. La mobilité temporelle réduite conduit pour certaines générations à l'inaccessibilité de certains biens. Il s'agit alors de se demander ce que les théories de la justice en général, et différentes interprétations de l'approche lockéenne en particulier, en tirent comme implications. Cela importe pour déterminer la capacité d'une théorie intergénérationnelle à appréhender la succession de périodes favorables et défavorables dans l'histoire. Nous pensons avoir illustré dans l'espace intergénérationnel les limitations d'une approche lockéenne de type antidistributif. Et nous avons montré que l'interprétation lockéenne «neutre» peut nous conduire à une clause lockéenne dont le contenu est nettement plus plausible, même s'il est éventuellement moins authentiquement lockéen. Nous pourrions d'ailleurs répéter l'exercice avec une théorie néohobbesienne en nous demandant si nos obligations de justice envers un tiers seraient d'autant plus faibles qu'il aurait une capacité plus limitée à nous nuire, y compris physiquement (plutôt que de nous priver simplement de 
ressources externes). Et plus généralement, nous pourrions nous demander si cette mobilité temporelle réduite a des conséquences pour d'autres théories de la justice. Pour l'égalitarisme, l'inaccessibilité de biens situés dans d'autres périodes ne semble pas avoir les mêmes conséquences, même si une plus ample recherche serait probablement nécessaire sur ce point.

\section{Enclaves temporelles et transmission extra-périodique des ressources}

I3. Nous nous sommes centrés jusqu'à présent sur le statut de certains biens dans la mesure où ils nous seraient inaccessibles en raison du caractère situé de notre naissance et de la nature limitée de notre longévité. Cela réduit notre capacité à y accéder et à consommer nous-mêmes ces biens. Nous voudrions à présent prendre les choses par un autre bout et nous demander ce que notre mobilité temporelle réduite implique quant à notre capacité de transmettre certains biens (du gaz naturel, une culture amérindienne, une langue en déclin, une technologie de pointe, etc.) à des générations avec lesquelles nous n'interagissons pas. Si les ressources éphémères ne peuvent être transmises au delà de leur période d'existence, d'autres ressources peuvent l'être. Si la consommation de biens strictement extra-périodiques est impossible, il n'en va pas de même de la consommation de biens durables. Mais pour que ces biens durables arrivent jusqu'à nous, ils doivent nous être transmis. C'est là qu'intervient la question de la transmission extra-périodique.

Si un certain nombre de pays dans le monde sont enclavés et tributaires, pour atteindre d'autres territoires, d'un accès à la mer qui dépend du bon vouloir d'un pays de transit, cette situation est généralisée dans le cas des générations. En effet, toute génération est enclavée, même si elle est à la fois une génération enclavée dans sa période ("period-locked»), dépendant de la suivante pour transférer ce qui lui importe aux générations futures, et une génération de transit en charge du patrimoine transmis par les générations précédentes. En outre, la situation est non seulement généralisée mais aussi radicalisée. Si, dans le cas des territoires enclavés, il existe souvent des territoires de transit alternatifs au cas où l'un d'eux refuserait le passage, l'unidirectionalité du temps est telle que, dans le cas des générations, la génération suivante est un passage obligé ${ }^{15}$. Et toute transmission au delà de la génération qui nous suit directement dépendra d'ailleurs de plusieurs générations de transit. Quelles sont alors les implications de notre quasiimmobilité générationnelle pour la transmission de tels biens au delà de notre période d'existence? Et en quoi cela importe-t-il pour une théorie de la justice?

La question de la transmission de biens au-delà de notre période d'existence comporte deux volets. D'une part, il y a celui de la transmission vers le passé, à rebours, sur laquelle nous reviendrons dans la troisième partie de 
cet article. Et d'autre part, il y a la transmission vers le futur, sur laquelle nous nous penchons ici. Pour le dire de manière un peu succinte, si la transmission vers le passé est impossible, ce n'est pas le cas de la transmission vers le futur, en tout cas pour de nombreux biens. Mais ce qui est intéressant, c'est que cette transmission vers le futur nous invite à bien penser la nature des obligations de chaque génération à cet égard, en tant que génération de transit.

I4. Dans la section précédente, nous nous sommes intéressé à l'impossibilité, pour les habitants d'un territoire insulaire, d'atteindre un autre territoire insulaire en raison de la conjonction d'obstacles internes et externes tels que l'hostilité du milieu marin, l'indisponibilité de ressources naturelles pour construire des bateaux capables d'affronter la haute mer, l'incapacité de s'y rendre à la nage, l'absence de technologie navale suffisante. Dans le cas des générations, la nature de l'obstacle peut être analysée plus directement comme le fruit des limites de nos ressources internes. Concentronsnous cependant un instant sur le rôle de l'océan. Dans le cas des ressources endémiques, la mer était un obstacle à l'atteinte d'un autre territoire. Pourtant, dans le cas de territoires constituant un continent, l'accès à la mer peut aussi permettre de contourner l'obstacle que constitue un territoire pour en rejoindre un troisième. Dans le premier cas, les résidents de $T_{1}$ sont confrontés à l'océan dans leur désir de rejoindre $\mathrm{T}_{2}$. Dans le second cas, les résidents de $T_{1}$, pourvus cette fois de navires, profitent de leur accès à la haute mer pour rejoindre $\mathrm{T}_{3}$ sans passer par $\mathrm{T}_{2}$. Cela n'est cependant pas possible si $\mathrm{T}_{1}$ est un territoire enclavé - du latin inclavatus qui signifie "enfermé à clef " -, sans accès à la mer. Notre enclavement générationnel consiste de la même manière dans le fait que, pour atteindre une période $\mathrm{P}_{3}$ (occupée par les générations futures), nous sommes forcés de passer par la période $P_{2}$ (occupée par les générations limitrophes suivantes).

Les situations d'enclavement sont telles qu'elles engendrent une dépendance entre territoires/périodes enclavés et territoires/périodes de transit. Mais qui dit "dépendance» dit que nous devons concevoir les générations de transit comme des obstacles, mais aussi comme des alliés (sans elles, pas de transmission possible pour la plupart des biens immatériels); comme les résidents de périodes que devra traverser le patrimoine transmis, mais aussi comme des passeurs; comme des murs, mais aussi comme des ponts. Et cela appelle au moins deux types de réflexions.

La première a trait à la nature de cette dépendance et à l'importance de penser en conséquence l'articulation de la transmission d'un patrimoine et les obligations passives, mais aussi actives, qu'elle engendre pour chaque génération. Nous pensons par exemple souvent la métrique de ce que nous transmettons en termes de stock. Mais il est essentiel de bien l'articuler avec l'effort qu'implique la préservation de ce stock, ou la compensation de sa dégradation. Le second type de réflexion a trait aux stratégies visant à 
réduire notre dépendance par rapport aux générations de transit dans cette transmission, et à la mesure dans laquelle ces stratégies de rigidification (architecturale, constitutionnelle, etc.) sont nécessaires et/ou acceptables. Envisageons chacun de ces deux aspects successivement.

I 5. Ce n'est pas l'objet du présent article d'examiner la nature des obligations légales des pays de transit envers les pays enclavés, telles qu'elles ressortent des nombreux traités bilatéraux existants qui relèvent du droit de la mer. Mais on peut conjecturer que les obligations imposées aux pays de transit en vue de garantir l'accès à la mer des pays enclavés sont principalement des obligations passives, de laisser passer, et des obligations qui concernent principalement le transport de biens matériels. L'hypothèse est ici que, dans le cas des générations, la part de la dimension active des obligations des pays de transit est bien plus importante, tant pour les biens matériels que pour les biens immatériels. On peut distinguer à cet égard les tâches de transport (biens matériels) et celles de transmission (biens immatériels) ${ }^{16}$.

Dans la relation entre un pays enclavé et un pays de transit, on demandera par exemple au pays de transit de rendre disponible une voie de communication, ce qui représente bien sûr un coût. Il est cependant tout à fait concevable que les autres coûts de transport qui ne concernent pas l'infrastructure restent à charge du pays enclavé lui-même. Il peut acquérir ses propres camions, disposer de ses propres chauffeurs et les faire traverser eux-mêmes le pays de transit. Dans le cas des générations, les "chauffeurs» appartiennent nécessairement à la génération de transit elle-même. La part active liée au «transport» doit nécessairement être prise en charge par la génération de transit, contrairement à ce qui se passe pour les pays enclavés. En outre, dans l'espace des générations, les coûts de "transport " peuvent être caractérisés comme des coûts de stockage. Et une comparaison fine de l'acte de transport et de l'acte de stockage permettrait sans doute d'identifier de plus amples différences possibles entre attentes de justice intergénérationnelle et attentes de justice globale. Mais dès que le stockage concerne un bien matériel qui se dégrade, il est évident que ce stockage doit être pensé de concert avec des obligations d'entretien. Même pour les biens matériels, on va donc au-delà d'un "droit de passage» temporel. Ajoutons aussi que la distinction entre obligations passives et obligations actives ne coïncide pas avec une distinction entre des efforts moins significatifs et des efforts plus significatifs. Les coûts d'opportunité d'une obligation de ne pas faire - par exemple quand des monuments, des cimetières, etc., occupent un espace qui pourrait être affecté à d'autres activités — peuvent être tout aussi significatifs que ceux associés à certaines obligations de faire. Pour prendre un

16. Certains des éléments discutés ici sont le fruit d'interactions avec Jacques Thisse que je remercie. 
exemple dans un autre domaine, il nous en coûte parfois plus de nous taire que de parler.

Il ne s'agit cependant pas seulement de transporter ou de stocker des biens matériels, mais aussi de transmettre de la technologie, une culture, ce qui passe par l'éducation dans ses formes les plus diverses. Or, cela est central pour plusieurs raisons. D'abord, parce que si des obligations de faire existent dans le cas de la transmission des biens matériels, elles sont encore plus significatives dans celle des biens immatériels. Ensuite, parce que la transmission (de connaissances) peut fonctionner comme substitut au stockage (de biens matériels). Plutôt que de stocker physiquement un artefact, on peut, à travers la transmission de connaissances, en assurer la reproduction ultérieure. Plutôt que de congeler par exemple des statues de glace pendant des décennies, on peut en garantir la reproductibilité (et le renouvellement) en apprenant aux nouvelles générations à sculpter la glace. On peut donc transmettre le «mode d'emploi », la capacité de le lire et celle de l'exécuter, au lieu d'investir dans des coûts de stockage du bien matériel.

En outre, l'on peut aussi avancer l'idée selon laquelle la technologie prend une place de plus en plus importante dans ce qui est ou doit être transmis au cours de l'histoire, à la fois en raison de son propre développement, mais aussi en raison de l'épuisement progressif des ressources naturelles non renouvelables auxquelles doivent se substituer une part de plus en plus grande de ressources immatérielles. Cela signifie aussi que la métrique d'une théorie de la justice, la définition de son distribuendum, doit prendre en compte - lorsqu'il s'agit de déterminer si nous transmettons plus ou moins à nos descendants que ce que nous ont légué nos parents - le fait que le potentiel productif de certains actifs transférés soit plus ou moins exigeant en travail. Une ressource naturelle inerte peut exiger ainsi moins de travail de la part de la génération de transit - pour ce qui est de son transport intergénérationnel - que n'en exigerait une technologie pour sa transmission intergénérationnelle. Or un surplus de travail peut engendrer une perte de bien-être significative de la génération de transit si celle-ci est elle aussi préoccupée par la satisfaction de ses propres obligations de justice envers ses descendants. L'idée ici n'est pas tant qu'il faudrait rémunérer la génération d'aval pour son travail ${ }^{17}$. C'est plutôt qu'une génération d'amont doit prendre en compte dans l'évaluation du caractère juste de la composition et de la quantité de ce qu'elle transfère le travail que ce patrimoine ainsi légué imposera aux générations de transit successives.

Enfin, la transmission n'est pas qu'un substitut au stockage. Elle concerne aussi autre chose que la production de biens matériels. La transmission d'une culture permet par exemple de comprendre la logique des institutions chargées de gérer les relations entre les personnes constituant une société. Et elle est porteuse de l'ensemble de notre rapport au monde.

17. Nous répondons ici à une suggestion d'un des évaluateurs anonymes. 
Cette dimension, absente des préoccupations du droit de la mer quand il aborde le problème des pays enclavés, est tout à fait centrale pour la justice intergénérationnelle.

I6. Notons à ce stade qu'une théorie lockéenne - pour reprendre le même exemple - aura tendance, dans certaines de ses versions, à traiter les biens culturels et naturels de manière distincte, les obligations lockéennes portant principalement sur ces derniers, alors que les biens culturels constituent en réalité une part majeure de la valeur de nos «avoirs». En outre - et cela n'est pas entièrement indépendant du premier point - une théorie lockéenne aura tendance à se centrer aussi plutôt sur les obligations passives («laisser la planète...»), alors que mettre l'accent sur notre dépendance générationnelle nous permet d'insister sur le fait que les obligations de faire sont déterminantes, en particulier pour la transmission de notre patrimoine immatériel. De manière générale, une théorie qui tend à ne pas dépasser des obligations de ne pas faire aura donc du mal à proposer une théorie de la justice intergénérationnelle convaincante. Notons aussi qu'une théorie de la justice qui accorderait beaucoup de place à l'incertitude devrait prendre en compte le fait que cette dépendance inter-générationnelle engendre le risque qu'un patrimoine patiemment accumulé au fil du temps puisse être totalement détruit par la négligence d'une seule génération intermédiaire future, ce qui pourrait constituer une des justifications envisageables d'un taux d'actualisation non nul.

I7. Notre dépendance envers les générations intermédiaires futures pour le transfert des biens et la transmission de notre patrimoine technologique et culturel aux générations plus éloignées requiert ainsi la définition d'obligations des générations de transit, dont nous faisons tous partie. Mais cette dépendance peut donner lieu à une seconde stratégie, dite de rigidification, qui vise à réduire la marge de manœuvre des générations intermédiaires et à tenter de garantir un peu mieux le respect de certains principes ou de certains biens.

Les stratégies de rigidification peuvent prendre plusieurs formes: une génération peut vouloir accorder un régime particulier à certaines règles de droit (constitutionnalisation), garantir une rigidité particulière à certains édifices «en dur» (architecture), réduire la réversibilité du mode de stockage de déchets nucléaires, voire - dans une forme plus radicale - faire disparaître «à jamais » certaines menaces (ex: suppression d'une souche virale). La rigidification peut aussi servir des objectifs maléfiques ou être simplement l'alliée des négligences d'une génération, comme dans le cas des multiples bombes à retardement, au sens propre (ex: mines anti-personnel) comme au sens figuré (ex: régulation insuffisante des marchés financiers), que nous amorçons de notre vivant, en veillant parfois délibérément à ce qu'il soit très difficile pour les générations intermédiaires de les désamorcer. 
S'il n'est pas possible d'entrer dans les détails, signalons cependant que ce type de stratégie de rigidification n'a pas vraiment d'équivalent en droit international dans le cas des pays enclavés, la multilatéralisation n'en étant qu'une approximation. Insistons aussi sur les limites d'une telle stratégie: rigidifier une règle en la constitutionnalisant ne met pas à l'abri par exemple d'un changement révolutionnaire de constitution. Mais ce qui nous intéresse surtout, c'est d'identifier la nature de la difficulté que pose une telle stratégie de rigidification. Et là, la comparaison avec les nations est tout à fait appropriée puisque le problème peut effectivement être analysé comme un problème de souveraineté, générationnelle en l'occurrence. Nous avons exploré ailleurs ce diagnostic et examiné aussi dans quelle mesure une telle stratégie peut être défendue ${ }^{18}$.

I8. En somme, cette approche centrée sur la comparaison avec les pays enclavés nous a permis de mettre deux choses en exergue, qui sont en réalité deux différences très significatives entre nations et générations. D’abord, notre dépendance à l'égard de nos successeurs pour le transfert et la transmission des éléments patrimoniaux qui nous tiennent à cœur, et en particulier pour leur part immatérielle, nous a invité à mettre l'accent sur les obligations de faire qui incombent à chaque génération de transit, et qui sont beaucoup moins fortes dans le cas des territoires de transit. Toute théorie de la justice entre les générations doit, dans la définition de sa métrique, prendre ce fait en considération. Cela a en particulier son importance dans la définition du distribuendum. Ensuite, nous avons pointé les stratégies de rigidification qu'une génération peut mettre en œuvre pour atténuer cette dépendance, un élément qui, lui non plus, n'est pas présent dans la même mesure dans le cas des nations.

\section{III. Écoulement du temps et asymétrie des vulnérabilités mutuelles}

I9. Les deux sections précédentes ont traité, chacune à leur manière, des conséquences des limites à la mobilité temporelle des personnes et à la transmissibilité temporelle des biens. Il n'en ira pas autrement dans la présente section, même si nous allons à nouveau envisager les choses sous un angle différent et partir d'une autre analogie spatiale. Si, dans la première section, nous nous sommes concentré sur la question de la consommation extrapériodique des biens, dans la seconde, nous nous sommes intéressé à la transmission extra-périodique de ces mêmes biens. Cette troisième section s'intéresse ici aussi à la transmission des biens (et des maux), mais non plus sous l'angle de notre dépendance envers les générations intermédiaires. Nous allons plutôt nous intéresser aux conséquences, pour une théorie de la justice et une théorie de la démocratie, de notre incapacité à transmettre des biens à rebours. 
L'analogie appropriée à cet égard est celle d'un fleuve. Elle est imparfaite et elle n'est pas neuve ${ }^{19}$ mais elle reste potentiellement riche. L'élément central de cette analogie tient évidemment à la direction de l'écoulement de l'eau. Mais d'autres aspects pourraient être explorés également. Pensons au fait qu'au fur et à mesure de son parcours le débit du fleuve fluctue, la quantité d'eau étant généralement croissante ${ }^{20}$ et la vitesse d'écoulement n'étant pas constante. Nous nous limiterons cependant ici à la direction de l'écoulement. À nouveau, si ce phénomène d'unidirectionnalité (ou de non-transmissibilité inverse) est présent dans divers cadres internationaux, notamment dans celui des fleuves, il est systématique dans le cas des générations, une fois dépassée la période d'enchevêtrement, de chevauchement. Et nous verrons plus bas que, même en comparaison avec les fleuves eux-mêmes, l'unidirectionnalité est plus radicale dans l'espace générationnel que dans l'espace fluvial.

20. Avant d'entamer l'examen des questions normatives clef liées à cette analogie, quelques mots sur la structure temporelle des coûts et bénéfices. Une activité donnée peut générer soit une répartition temporelle uniforme des coûts et des bénéfices (distribution uniforme), soit des coûts concentrés au début, avec des bénéfices plus tard (distribution chargée en amont), soit encore des bénéfices immédiats et des coûts repoussés vers le futur (distribution chargée en aval) ${ }^{21}$. À titre d'illustration, pensons respectivement à la mise en place d'une constitution qui bénéficie à la fois à la génération actuelle et aux générations futures sans coûts d'adoption significatifs, à des recherches pharmaceutiques de longue haleine qui ne porteront leurs fruits que dans plusieurs décennies, ou à des centrales nucléaires dont l'activité est limitée à quelques décennies mais dont les déchets devront être suivis pendant bien plus longtemps.

L'idée à garder à l'esprit est alors la suivante: le fait qu'un contexte unidirectionnel génère une structure de pouvoir asymétrique où l'acteur d'amont a plus de pouvoir sur l'acteur d'aval que l'inverse, ne va pas toujours de pair avec une distribution des coûts et bénéfices où les bénéfices seraient concentrés sur l'amont et les coûts sur l'aval. L'hypothèse - à discuter - selon laquelle, historiquement, nous serions aujourd'hui dans une situation plus avantageuse qu'il y a ro०० ans illustre ce point. Les tenants d'une telle hypothèse n'en concluraient pas pour autant que les générations d'il y a un millénaire avaient moins de pouvoir sur nous que nous n'en avons sur elles. On veillera d'ailleurs aussi à appréhender la distribution des coûts de manière suffisamment large, en y intégrant les bénéfices futurs qu'une pollution durable peut éventuellement rendre possible.

19. Barry (I977).

20. Merci à Chloé Castagnet d'avoir attiré mon attention sur ce point.

21. Gardiner (2006), Gosseries (2008). 
2I. Cette précision sur la distinction entre, d'une part, distribution des coûts et bénéfices d'un acte ou d'une omission et, d'autre part, distribution intertemporelle du pouvoir étant faite, soulignons d'abord brièvement l'importance de la direction d'écoulement du temps pour les théories de la justice. On peut la pointer, à titre d'illustration, pour trois approches très différentes de la justice. Envisageons d'abord une théorie centrée sur la réciprocité indirecte. Elle justifie l'existence d'obligations envers les générations suivantes en raison du fait que nous ayons reçu quelque chose des générations précédentes. Pour une telle théorie, l'impossibilité d'une réciprocation directe au bénéfice des générations précédentes - impossibilité qui résulte de l'unidirectionnalité du temps - est un ressort central. Sans une telle impossibilité, une telle logique serait incapable de justifier des obligations envers les générations suivantes ${ }^{22}$.

Envisageons ensuite une théorie centrée sur l'avantage mutuel. L'idée n'est plus ici de concevoir la justice comme l'absence de transferts nets (comme c'est le cas d'une théorie centrée sur la réciprocité), mais comme la distribution juste du surplus coopératif, du gain collectif lié à la coopération. Dans l'espace des générations, il y a clairement un surplus coopératif. Nous nous appuyons sur les efforts de nos prédécesseurs pour construire encore et encore. Mais la difficulté est de savoir si ce bénéfice peut être mutuel. Si une coopération intergénérationnelle engendre des bénéfices qui ne peuvent être partagés parce qu'ils sont irrémédiablement concentrés sur les générations ultérieures, cela menace non seulement une distribution juste de tels bénéfices, mais plus radicalement encore, pour les tenants de cette logique, l'existence même d'obligations envers les générations futures.

C'est cette impossibilité de bénéficier aux générations passées, cette non-transmissibilité des bénéfices à rebours qui est centrale aussi pour Rawls, même si on s'écarte d'une logique coopérativiste de l'avantage mutuel pour se concentrer sur une approche impartialiste. Cette difficulté se traduit à divers niveaux. En voici deux illustrations. D'abord, Rawls abandonne le maximin dans l'espace des générations parce qu'il affirme que «c'est un fait naturel que les générations soient distribuées dans le temps et que les bénéfices effectifs ne s'écoulent que dans une direction. Cette situation ne peut être modifiée et la question de justice ne se pose donc $\mathrm{pas}^{23}$ ». Nous avons détaillé ailleurs la manière de répondre à cette difficultée ${ }^{24}$. Ensuite, pour une théorie préoccupée du sort du plus défavorisé, la difficulté à justifier une accumulation est sans doute liée en partie aussi à l'impossibilité des bénéfices à rebours au delà de l'enchevêtrement. On pourrait penser que les choses sont moins claires ici parce que c'est l'exigence d'accumulation qui, au premier chef, semble faire que les premières générations - plus

22. Voir Gosseries (2009).

23. Rawls (I97I/I999, 254) (notre traduction).

24. Gaspart et Gosseries (2007), Gosseries (20I4a). 
défavorisées - se retrouveront dans une situation moins favorable que celle qui aurait pu être la leur en cas d'accumulation. Mais c'est oublier la chose suivante: reporter la consommation au profit de l'investissement productif ne devrait pas nécessairement impliquer une accumulation nette si les fruits de l'investissement pouvaient être uniformément redistribués à l'ensemble des générations impliquées. Et c'est l'impossibilité d'une telle redistribution qui fait que l'on ne peut accroître le gâteau intergénérationnel sans placer les générations précédentes dans une situation pire que la notre, et dans une situation pire que celle qui aurait pu être la leur si elles ne participaient pas à l'effort d'investissement intergénérationnel.

22. Comme on le voit, la question de l'unidirectionnalité du temps est déterminante pour une série de théories de la justice. Et on peut même formuler l'hypothèse selon laquelle elle a des impacts pour l'ensemble de ces théories. Ce sur quoi je voudrais cependant me pencher à présent, tout en restant fidèle à l'analogie fluviale, c'est l'impact de cette unidirectionnalité pour une théorie de la démocratie, et en particulier pour une dimension limitée mais importante d'une telle théorie: l'allocation du droit de vote et la détermination de son poids. De quelle manière faut-il concevoir le poids de vote de chaque génération?

Si l'on prend au sérieux l'idée de souveraineté générationnelle promue par Jefferson ${ }^{25}$, chaque génération devrait en principe avoir les pleins pouvoirs sur les politiques qui l'affectent. Dans un monde où les choix d'une génération seraient sans impact sur les conditions d'existence de la suivante, l'on pourrait peut-être se satisfaire d'une telle approche. Dans un tel monde, sans externalités extra-périodiques et où chaque génération serait mise au monde par des cigognes, l'idée même d'une rigidité constitutionnelle qui prétendrait se prolonger au delà de l'existence de la génération constituante serait probablement plus difficile à justifier ${ }^{26}$. Notre monde est cependant riche d'externalités territoriales, fluviales ou extra-périodiques. Si une nation riveraine d'amont pollue le fleuve, la nation riveraine d'aval en subira les conséquences. Il en va de même des générations. Dans un monde anarchique, la distribution du pouvoir effectif et des vulnérabilités entre générations est donc la suivante: la génération d'amont a plus de pouvoir sur la génération d'aval que l'inverse; la vulnérabilité de la génération d'aval par rapport à la génération d'amont est plus forte que l'inverse.

Comment appréhender cette asymétrie de pouvoir de fait et en tenir compte dans la détermination du poids de vote tel qu'il devrait être alloué à chaque nation riveraine ou à chaque génération successive dans un régime qui se voudrait le plus démocratique possible? Dans la littérature récente consacrée à la définition du demos - à savoir le groupe de personnes qui

25. Gosseries (20I4b).

26. Ibid. 
doit se voir reconnaitre le droit de vote par rapport à un problème donné un des critères proposé est le "all affected principle ${ }^{27}$ ». L'idée est que mon inclusion dans le demos ne doit pas être déterminée, par exemple en fonction de l'existence et de l'ampleur de la contribution d'une personne à la collectivité (dans l'esprit de "no tax, no vote»). Elle doit plutôt l'être en fonction du fait qu'une personne est potentiellement affectée par les décisions de cette communauté (dans l'esprit de "no tax without representation»), et du degré auquel elle l'est. Ce critère présente une série de difficultés significatives. D'une part, le contenu même de la définition, et donc l'identité et la manière dont les groupes impactés seront affectés variera en fonction du groupe chargé de prendre la décision, ce qui rend le critère relativement instable dans ses applications. D'autre part, ce que l'on entend pas être "affecté" requiert une définition. Cela étant, on perçoit la logique générale du principe.

23. Une traduction possible de ce principe du «all affected» consiste à en faire une lecture proportionnaliste, ${ }^{28}$ dépassant ainsi la logique du «one (wo)man, one vote». Il s'agit alors d'ajuster le poids de vote au degré auquel les personnes sont potentiellement affectées. Envisageons successivement deux exemples, l'un non fluvial, et l'autre fluvial. D'une certaine manière, la décentralisation ou la fédéralisation d'un État peut être lue comme une tentative de traduire une lecture proportionnaliste du principe. Ainsi, un citoyen de Rimouski a le même poids de vote qu'un citoyen de Schefferville aux élections provinciales. Mais il aura plus à dire que le résident de Schefferville aux élections municipales de Rimouski, le résident de Schefferville n'y ayant rien à dire du tout. On peut le justifier par le fait qu'un résident de Schefferville sera moins (et ici, "pas du tout») affecté par les décisions locales des autorités de Rimouski que le résident de Rimouski lui-même. Bien sûr, dans cet exemple territorial standard, si le résident de Rimouski a plus à dire à Rimouski, le résident de Schefferville a plus à dire à Schefferville aussi. La situation est donc symétrique, et le pouvoir de vote est réparti de manière discrète ( $\mathrm{I}$ ou o) plutôt que continue, ce qui rend peut-être moins visible le caractère proportionnel du pouvoir accordé à chacun de ces deux citoyens.

Ce caractère proportionnel devient plus visible si l'on considère la mise en place d'une autorité de gestion du fleuve Saint-Laurent. Remplaçons dans ce cas Schefferville par la municipalité de Québec, située en amont de Rimouski, le long du même fleuve Saint-Laurent. La lecture proportionnaliste de la règle précitée d'inclusion dans le demos exigerait ici que Rimouski bénéficie de garanties particulières par rapport à Québec dans un processus multilatéral de décision, grâce à une surpondération de vote, voire un droit de veto pour certains types de décisions concernant le fleuve, par exemple la

27. Voir par exemple Goodin (2007).

28. Brighouse et Fleurbaey (2008). 
qualité de son eau ou son débit. En effet, l'on peut postuler que la capacité de Québec de nuire, par ses décisions, à Rimouski, est sans doute plus forte que l'inverse. Mais cet exemple montre aussi que l'unidirectionnalité est plus forte dans le monde des générations que dans celui des fleuves, puisqu'il est possible de remonter un fleuve par exemple. Rimouski pourrait ainsi nuire fortement aussi à l'économie de Québec en empêchant les bateaux de remonter le Saint-Laurent. Le principe "all affected» exigerait alors des garanties particulières pour Québec, lorsque des décisions sont à prendre sur des questions de circulation sur le fleuve, plutôt que sur des question de qualité des eaux par exemple. On note aussi que si Rimouski est plus vulnérable aux décisions de Québec que l'inverse pour ce qui est de la qualité et du débit de l'eau, Québec se trouve dans la même situation par rapport à d'autres municipalités en amont, telles que Montréal.

24. La lecture proportionnaliste du principe «all affected» a le mérite de permettre une lecture unifiée d'une série d'intuitions que l'on peut avoir par rapport aux exigences d'une démocratie, et en particulier par rapport à la question de la nécessaire inclusivité électorale d'un tel régime. Elle permet sans doute aussi de relire les droits des minorités à l'aune de ce principe. Cela nous conduit à proposer un principe de distribution du poids de vote entre générations qui tient compte du caractère asymétrique de leur vulnérabilité mutuelle: une génération postérieure étant plus susceptible d'être affectée par les décisions d'une génération antérieure que l'inverse, un régime de décision collective devrait accorder aux générations postérieures un pouvoir plus important qu'aux générations antérieures.

On peut alors comprendre l'ampleur de la difficulté posée par les modes de décision intergénérationnels que nous connaissons. En pratique, les générations antérieures prennent seules les décisions. On pourrait croire que cela pose problème parce que l'exercice du pouvoir devrait être également partagé entre générations antérieures et générations postérieures. Ce serait manquer le caractère aggravé du diagnostic que révèle la conjonction d'une prise en considération de l'unidirectionnalité temporelle et d'une lecture proportionnaliste du principe de définition du demos. Le pouvoir est exercé dans les faits exclusivement par la génération antérieure alors qu'il devrait être exercé non pas également mais inégalement, avec un poids plus important pour la génération postérieure lorsque des décisions ayant un impact intergénérationnel sont en cause. Cela éclaire d'une lumière nouvelle la nature de l'asymétrie de pouvoir dans les processus de décision intergénérationnels, en montrant que la situation est pire qu'on ne le pense généralement au regard des exigences d'une théorie de la démocratie.

25. Je voudrais à présent conclure cette section par le bref examen de deux articulations plus délicates à effectuer, et qui, chacune, pointent vers des éléments qui rendraient éventuellement la génération antérieure plus vulnérable 
que la génération postérieure, atténuant l'effet de l'unidirectionnalité du temps.

D'abord, si l'on peut considérer une génération postérieure comme plus vulnérable aux décisions de l'antérieure que l'inverse, on doit aussi envisager la situation où la génération postérieure serait en même temps plus riche que l'antérieure. Accorder plus de pouvoir à la génération postérieure, outre la difficulté à approximer cela en pratique, peut donc conduire aussi à accorder plus de pouvoir aux plus riches. Et si la vulnérabilité d'une personne peut se penser comme une vulnérabilité par rapport aux actes d'autrui, elle peut aussi être évaluée en fonction des moyens matériels dont une personne dispose pour faire face à des décisions qui lui seraient défavorables. Une notion élargie de vulnérabilité qui prendrait en considération l'accumulation rendue possible par le passage du temps pourrait donc amener éventuellement à une lecture du principe «all affected " très différente de celle que nous venons proposer.

L'autre précision a trait à la relation entre la vulnérabilité d'une génération postérieure par rapport aux conséquences des décisions d'une génération antérieure, et la dépendance d'une génération antérieure par rapport à la bonne volonté des générations postérieures dans la transmission du patrimoine destiné à l'ensemble des générations à venir. Ici aussi, cela atténue l'asymétrie de vulnérabilité liée au caractère unidirectionnel du temps. En un sens, on peut dire que la génération antérieure a le «dernier mot» sur ce qu'elle va nous transmettre. Mais nous avons aussi le «dernier mot» sur ce que nous allons transmettre de ce que nous avons hérité d'elle. Et ce qui restera de notre héritage après notre mort peut importer beaucoup pour certains d'entre nous. Ici aussi, la vulnérabilité n'est pas localisée de manière aussi systématique dans le futur.

26. Que conclure de cette troisième section? Que l'unidirectionnalité du temps détermine le caractère asymétrique de la vulnérabilité mutuelle des générations. Cette asymétrie est centrale pour une lecture proportionnaliste $\mathrm{du}$ "all affected principle», dont l'objet est de déterminer quel poids de vote devrait être alloué à différents individus dans le cadre d'un processus de décision démocratique. Comme on peut l'imaginer, les différences d'analyse se jouent ici sur deux fronts. Sur la question de savoir si nous n'avons pas tendance à exagérer l'ampleur d'une telle asymétrie des vulnérabilités, non seulement dans le cas des fleuves, mais surtout dans celui des générations, spécialement si l'on prend au sérieux d'autres sources de vulnérabilité comme nous venons de le faire. Et sur les implications exactes - pour ce qui est de la démocratie et de la justice - d'une telle asymétrie en tant qu'elle porte non seulement sur notre vulnérabilité mutuelle dans l'espace intergénérationnel, mais aussi sur notre capacité à générer des bénéfices intergénérationnellement mutuels. 


\section{Conclusion}

27. Notre aventure intergénérationnelle ne ressemble ni à un fleuve tranquille ni à une mer d'huile. Les défis auxquels nous faisons face sont majeurs, sur le plan théorique, et plus encore sur le plan pratique. Notre incapacité à faire face au réchauffement climatique est abyssale. Et les difficultés que nous éprouvons plus généralement à limiter l'épuisement de nos ressources ou à rendre notre monde plus juste sont tout aussi préoccupantes. Si la philosophie politique peut nous aider, c'est en nous permettant de mieux percevoir la nature des enjeux et d'articuler les tenants et aboutissants des solutions possibles, mettant à jour, inlassablement, les intuitions normatives qui les sous-tendent. Il nous faut aussi constamment réinventer de nouveaux langages, à chaque fois que c'est nécessaire, à chaque fois que nous nous sommes accoutumés à un langage qui ne nous parle plus, à chaque fois que le langage que nous parlons nous empêche de voir ce qui se présente devant nous. On pourrait ainsi peut-être s'exprimer en termes de souveraineté ou de discrimination pour appréhender les relations entre générations, beaucoup plus que nous ne le faisons aujourd'hui. Cela importe en ce qui concerne la justification des réponses à apporter, tant pour ce qui est de l'ethos que pour ce qui est des institutions, aux défis colossaux qui sont les nôtres face à l'avenir.

C'est de la volonté de reprendre la question intergénérationnelle à partir d'un langage nouveau qu'a procédé ce texte, dont l'objectif est de comprendre ce que des théories déterminées, qui nous sont familières, impliquent quant à ce que nous devons aux générations à venir. Nous avons montré que notre mobilité temporelle réduite, le caractère enclavé des générations et la direction de l'écoulement du temps ont chacun un impact sur le contenu de ce que nous devons à nos successeurs ou sur la façon dont il faudrait concevoir démocratiquement nos institutions. Nous avons présenté plus haut les conclusions spécifiques auxquelles conduisait l'examen analogique de ces trois caractéristiques de la question intergénérationnelle. Nous avons en particulier mis l'accent sur la nécessité de vérifier si la théorie que nous privilégions est bien capable de tenir compte des fluctuations, d'une période à l'autre, des ressources qui ne sont accessibles qu'à certaines des générations. Nous avons aussi souligné la nécessité de prendre pleinement en considération les obligations de faire des générations de transit dont nous faisons tous partie. Et nous avons insisté enfin sur le caractère aggravé du diagnostic relatif à la distribution intergénérationnelle du pouvoir dans nos institutions, à la lumière de l'asymétrie de notre vulnérabilité mutuelle et d'une lecture proportionnaliste du "all affected principle». Ces trois points particuliers sont rendus particulièrement visibles par les analogies maritimes et fluviales que nous avons explorées. Elles en ont permis à la fois l'exploration et la dramatisation à partir d'espaces plus familiers. Cela n'est évidemment qu'une modeste pierre à un édifice qui ne saurait se satisfaire ni de théorie désincarnée ni d'action irréfléchie. 


\section{Bibliographie}

Barry, B. «Justice Between Generations » in P. M. S. Hacker and J. Raz (eds), Law, Morality and Society, Oxford, Oxford University Press, I977.

—. "Justice as Reciprocity», in E. Kamenka and A. Eh-Soon Tay (eds), Justice, London, Edward Arnold Publishers, I979.

Brighouse, H., \& M. Fleurbaey. «Democracy and Proportionality », Journal of Political Philosophy, I8(2), 2008, I37-I 55.

Crispini, N. La Trace. Approche de Germaine et Robert Hainard, Genève, Slatkine, I996, I76 p.

Gardiner, S. «Protecting Future Generations : Intergenerational Buck-Passing, Theoretical Ineptitude and a Brief for a Global Core Precautionary Principle », in J. Tremmel (ed.), Handbook of Intergenerational Justice, Cheltenham, Edward Elgar, 2006, p. I48-I69.

Gaspart F. \& A. Gosseries. "Are Generational Savings Unjust? ", Politics, Philosophy \& Economics, 6(2), 2007, 193-217.

Goodin, R. "Enfranchising All Affected Principles, and Its Alternatives ", Philosophy and Public Affairs, 35 (I), 2007, 40-68.

Gosseries, A. "What Do We Owe the Next Generation(s)? ", Loyola of Los Angeles Law Review, 35(I), 200I, 293-354.

. «Radiological Protection and Intergenerational Justice», in G. Eggermont \& B. Feltz (eds.), 2008.

. Ethics and Radiological Protection, Louvain-la-Neuve, AcademiaBruylant, p. I67-195.

. "Three Models of Intergenerational Reciprocity", in A. Gosseries \& L. Meyer (eds.). Intergenerational Justice, Oxford, Oxford University Press, 2009, p. II9-I 46.

- . "Nations, Generations and Climate Justice», Global Policy, 5(I), 20I 4a, 96-IO2.

- "The Intergenerational Case for Constitutional Rigidity", Ratio Juris, 27(4), 20I 4 b, sous presse.

Otsuka, M. Libertarianism without inequality, Oxford, Clarendon Press, 2003, I $70 \mathrm{p}$.

Paine, Th. I796. Agrarian Justice.

Parfit, D. Reasons and Persons, Oxford, Clarendon Press, I984.

Rawls. I97I. A Theory of Justice (Rev.), Oxford, OUP, I999, 538 p.

Steiner, H. \& P. Vallentyne. "Libertarian Theories of Intergenerational Justice », in Intergenerational Justice (Gosseries A. \& L. Meyer eds.), Oxford, OUP, 2009, p. 50-76.

Stilz, A. "Nations, States and Territory ", Ethics, I 2I(3), 20I I, 572-60I.

Vallentyne, P. \& H. Steiner (eds.). Left-libertarianism and its Critics: The Contemporary Debate, Palgrave, 2000. 